\title{
Efectos secundarios de la medicación antipsicótica y calidad de vida en pacientes con esquizofrenia latinoamericanos
}

\author{
Side effects of antipsychotic medication and quality of life in Latin-American patients \\ with schizophrenia
}

\author{
Alejandra Caqueo-Urízar \\ Universidad de Tarapacá, Arica, Chile. \\ Alfonso Urzúa \\ Universidad Católica del Norte, Antofagasta, Chile. \\ Mar Rus-Calafell \\ Department of Social Psychiatry, Institute of Psychiatry, King’s College London, London, United Kingdom.
}

Rec (22 de febrero de 2016) Acept (10 de febrero de 2017)

\begin{abstract}
Resumen
El estudio describe la relación entre los efectos secundarios a la medicación y la calidad de vida en pacientes con esquizofrenia. La muestra contó con 253 pacientes de los Servicios Públicos de Salud Mental de Bolivia, Perú y Chile quienes fueron evaluados con la Escala Barnes de Acatisia (EBA); la Escala de Simpson-Angus (SAS) y el Cuestionario de Calidad de Vida (SQoL-18). Ambas escalas: EBA ( $\beta=-2,17$; $\mathrm{p}=0,019)$ y $\mathrm{SAS}(\beta=-1,97 ; \mathrm{p}=0,034)$ presentaron una relación significativa con la escala de calidad de vida. Variables sociodemográficas como la edad $(\beta=-2,17 ; p=0,032)$; la ocupación $(\beta=-4,12 ; p=0,046)$; el nivel educativo $(\beta=-7,14 ; p=0,005)$; la etnia $(\beta=-3,91 ; p=0,035)$ y variables clínicas como severidad del trastorno (PANSS) mostraron también un peso significativo en la calidad de vida ( $\beta=-6,35 ; p=\leq 0,01)$. Al igual que en países desarrollados, los efectos secundarios de los antipsicóticos tienen una influencia en la calidad de vida de los pacientes.

Palabras clave: Efectos secundarios; calidad de vida; Esquizofrenia; Latinoamérica.
\end{abstract}

\begin{abstract}
The study describes the relationship between antipsychotic drug side effects and quality of life in a sample of individuals suffering from schizophrenia. The sample included 253 patients' users of different Public Mental Health Services from Bolivia, Peru and Chile who were evaluated with the Barnes Akathisia Rating Scale (BARS), the Simpson-Angus (SAS) and the Schizophrenia Quality of Life short version questionnaire (SQoL-18). A statistical significant correlation was found between quality of life (SQoL-18) and total scores in the BARS $(\beta=-2,17 ; p=0,019)$ and SAS $(\beta=-1,97 ; p=0,034)$. Socio-demographic variables, such as age $(\beta=-2,17 ; p=0,032)$, education $(\beta=-7,14 ; p=0,005)$, occupation $(\beta=-4,12 ; p=0,046)$ and ethnicity $(\beta=-3,91$; $p=0,035$ ), were significant predictors of quality of life. Clinical symptoms (PANSS) also contributed significantly to the model $(\beta=-6,35 ; p=\leq 0,01)$. As in developed countries, the side effects of antipsychotics have an influence on patients' quality of life.

Key words: Side effects; quality of life; Schizophrenia; Latin-American.
\end{abstract}

\footnotetext{
Correspondencia: Alejandra Caqueo-Urízar, Escuela de Psicología y Filosofía. Universidad de Tarapacá. Avenida 18 de Septiembre 2222. Arica, Chile, Fono: 56-58-2205622, Fax: 56-58-2205818Email: acaqueo@uta.cl, alurzua@ucn.cl, maria.rus-calafell@kcl.ac.uk
} 


\section{Introducción}

Los pacientes con esquizofrenia presentan un estándar de vida significativamente más pobre que otros en su comunidad (Pinikahana, Hapell, Hope y Keks, 2002). Una serie de factores influyen negativamente en su calidad de vida, entre estos: ser varón (Browne, Lane, Gervin, Morris, Kinsella, Larkin y O'Callaghan, 1996; Caron, Mercier, Diaz y Martin, 2005); de mayor edad (Browne et al., 1996); desempleado (Hofer, Kemmler, Eder, Edlinger, Hummer y Fleischhacker, 2004; Marwaha y Jhonson, 2004), sin pareja (Salokangas, Honkonen, Stengard y Koivisto, 2001); con mayor número de hospitalizaciones (Browne, et al., 1996); con escaso apoyo social (Górna, Jaracz, Rybakowski y Rybakowski, 2008); mayor severidad del trastorno (Rudnick, 2001) y con una mayor presencia de efectos secundarios a la medicación (Awad y Hogan, 1994; Bobes, Garcia-Portilla, Bascaran, Saiz y Bousono, 2007). Entre estos se encuentran: el aumento de peso (Allison, Mackell y McDonnell, 2003; Strassnig, Brar y Ganguli, 2003); síntomas adversos (Ritsner, Gibel, Perelroyzen, Kurs, Jabarin y Ratner, 2004); síndrome metabólico (Meyer, Nasrallah, McEvoy, Goff, Davis, Chakos, Patel, Keefe, Stroup y Lieberman, 2005); disfunción sexual masculina (Olfson et al., 2005); acatisia (Hofer et al., 2004) y síntomas extrapiramidales (Browne et al., 1996; Xiang, Wang, Wang, Chiu, Zhao, Chen, Chan, Lee y Ungvari, 2010). Estos últimos síntomas han sido escasamente estudiados en países Latinoamericanos donde el acceso a los antipsicóticos atípicos no es generalizado para todo los pacientes, más aún, en algunos de estas regiones, el acceso a la medicación antipsicótica, es complejo.

El objetivo del estudio es describir la relación entre los efectos secundarios a la medicación y la calidad de vida en pacientes con esquizofrenia en tres países latinoamericanos.

\section{Método}

La muestra contó con 253 pacientes con diagnóstico de esquizofrenia que asisten a tratamiento ambulatorio en los Servicios Públicos de Salud Mental en La Paz, Bolivia $(32,8 \%)$; Tacna, Perú $(33,6 \%)$ y Arica, Chile $(33,6 \%)$. Los pacientes fueron evaluados con la Escala Barnes de Acatisia (EBA) (Barnes, 1989) que evalúa la presencia y gravedad de la acatisia inducida por fármacos a través de 4 ítems; la Escala para Evaluación de Síntomas Colaterales Extrapiramidales de Simpson-Angus (Simpson y Angus, 1970) de 6 ítems, puntuados de 0 a 4 y el Cuestionario de Calidad de Vida
(SQoL 18) (Boyer, Simeoni, Loundou, D`Amato, Reine, Lancon y Auquier, 2010) que para efectos de este estudio, solo se considerará su puntuación final.

Se realizarán análisis univariantes y multivariantes controlando las variables socio-demográficas y clínicas a través de la Escala para el Síndrome Positivo y Negativo de la Esquizofrenia (PANSS) (Kay, Fiszbein y Opler 1987).

\section{Resultados}

Doscientos cincuenta y tres pacientes con esquizofrenia participaron en el presente estudio. La edad media fue de 35,6 años, $66,4 \%$ eran hombres y $46,2 \%$ de los pacientes pertenecían a la etnia Aymara. Un 93,7\% de los pacientes no estaba casado ni tenía pareja, un $69,6 \%$ no tenía ocupación y un $84,2 \%$ presentaba bajo nivel educativo $(\leq 12$ años). La severidad de los síntomas fue moderada, con una puntuación total de PANSS de 71,3 (DT=28.2). La media en la Escala de Barnes (EBA) fue de $0,87(\mathrm{DT}=1,97)$ por debajo del puntaje de corte para el diagnóstico de acatisia. La media en la Escala de Simpson-Angus (SAS) fue de 0,31 $(\mathrm{DT}=0,41)$ por debajo también del umbral de presencia y gravedad de la sintomatología parkinsoniana. Finalmente la media del índice total de calidad de vida evaluado con el SQoL 18 fue de 54,3 (DT=14,4) de un rango de 0-100.

Los análisis univariantes entre la Escala de Barnes (EBA) y la Escala de Calidad de vida (SQoL 18) muestran una relación significativa $(\beta=-2,17 ; p=0,019)$, de igual forma es la relación entre Escala de Simpson-Angus (SAS) y la escala de Calidad de vida (SQoL 18) $(\beta=-1,97 ; p=0,034)$.

Los análisis multivariantes mostraron que la asociación entre los efectos secundarios y calidad de vida se mantuvo significativa para la Escala de Simpson-Angus (SAS) $(\beta=2,53 ; p=0,043)$, no así para la Escala de Barnes (EBA) $(\beta=-0,69 ; p=0,504)$. Variables sociodemográficas como la edad ( $(=-2,17 ; p=0,032)$ : la ocupación $(\beta=-4,12 ; p=0,046)$; el nivel educativo $(\beta=-7,14 ; p=0,005)$; la etnia $(\beta=-3,91$; $\mathrm{p}=0,035)$ y variables clínicas como severidad del trastorno (PANSS) mostraron un peso significativo en la calidad de vida $(\beta=-6,35 ; p=p \leq 0,01)$ (tabla 1$)$.

\section{Discusión}

$\mathrm{Al}$ igual que en países desarrollados, los efectos secundarios de la medicación antipsicótica tienen una influencia en la calidad de vida de los pacientes con esquizofrenia en 
Tabla 1. Modelos de regresión lineal

\begin{tabular}{ll}
\hline & Calidad de Vida (SQoL-18) \\
\hline Modelo No-ajustado & Coeficiente de regresión ß $(95 \%$ IC) \\
\hline Escala Barnes de Acatisia (EBA) & $-2.17 *(-3.99 ;-0.35)$ \\
Escala de Simpson-Angus (SAS) & $-1.97^{*}(-3.79 ;-0.14)$ \\
\hline Modelo Ajustado & \\
\hline Escala Barnes de Acatisia (EBA) & $-0.69(-2.74 ; 1.35)$ \\
Escala de Simpson-Angus (SAS) & $2.53^{*}(0.08 ; 4.99)$ \\
Sexo (Mujer/Hombre) & $-2.45(-6.45 ; 1.55)$ \\
Edad & $-2.17 *(-4.15 ;-0.19)$ \\
Estado Civil (Con pareja/sin pareja) & $-1.01(-9.08 ; 7.06)$ \\
Ocupación (Sí/No) & $-4.12 *(-8.17 ;-0.07)$ \\
Etnia (Aymara/No-Aymara) & $-3.91 *(-7.55 ;-0.27)$ \\
Nivel educativo (<12 años/ $\geq 12$ años) & $-7.14 *(-12.08 ;-2.20)$ \\
PANSS total & $-6.35^{*}(-8.61 ;-4.09)$ \\
\hline
\end{tabular}

Nota. B: Representa el cambio en la desviación típica del puntaje de Calidad de vida como resultado del cambio de una desviación típica en la variable independiente. ${ }^{*} \mathrm{p} \leq 0.05 ; * * \mathrm{p} \leq 0.01$.

PANSS: Escala para el Síndrome Positivo y Negativo de la Esquizofrenia.

estas regiones de Latinoamerica (Browne, et al., 1996). Las razones probablemente responden a las mismas observadas en otros contextos, esto es: la presencia de efectos secundarios de los antipsicóticos, genera en el paciente un grado de malestar que lo lleva a presentar una baja adherencia al tratamiento y en casos más graves, lo llevan a descontinuar el fármaco, con las consecuencias negativas que esto tiene en su bienestar (Lindström, 1994).

Los resultados muestran también que aquellos pacientes más jóvenes, desempleados, con bajo nivel educativo e indígenas presentan peor calidad de vida. Dichos factores están claramente relacionados con las condiciones socioeconómicas experimentadas por estos pacientes. Este hallazgo no es sorprendente en América Latina, considerando el informe de la Organización Mundial de la Salud (OMS) sobre los sistemas de salud mental, el cual reporta la ausencia de cobertura de seguridad social universal para los trastornos mentales, la falta de capacidad en algunos pacientes para la compra de medicamentos antipsicóticos y la limitada función de la atención primaria en el área de salud mental (WHO 2013). De hecho, una barrera importante a la asistencia de los Servicios de Salud Mental se relaciona con las dificultades en el acceso geográfico en una cantidad significativa de pacientes. Se ha demostrado una relación inversa entre la distancia o tiempo del recorrido a los centros clínicos y el uso de servicios de salud, especialmente para los pacientes crónicos, pobres y socialmente excluidos (Peters, Garg, Bloom, Walker, Brieger y Rahman, 2008).
Factores que influyen en la calidad de vida, como los encontrados en este estudio: desempleo, minoría étnica y bajo nivel educativo, refuerzan la idea de que los trastornos mentales y pobreza están estrechamente relacionados (Wilton, 2004; Krieger, 2007; Marmot, Friel, Bell, Houweling y Taylor, 2008). Lo que para algunos genera un círculo vicioso negativo "la pobreza engendra enfermedad mental y esta mantiene a la gente pobre, más pobre" (Wagstaff, 2002; Lund, De Silva, Plagerson, Cooper, Chisholm, Das, Knapp y Patel, 2011).

Este estudio presenta limitaciones, entre ellas, su diseño transversal no permite establecer relaciones causales. En segundo lugar, si bien se contó una muestra amplia, los resultados no pueden ser generalizados a la totalidad de la población de pacientes en Latinoamérica. Se necesitan estudios más amplios y con grupos más diversos para confirmar estos hallazgos. Finalmente, el estudio no contó con una clasificación del tipo de antipsicótico utilizado por los pacientes, futuras investigaciones deberían considerar diferencialmente entre los efectos de antipsicóticos clásicos y atípicos.

\section{Conclusiones}

Los efectos secundarios influyen en la calidad de vida de pacientes con esquizofrenia en estas regiones latinoamericanas. Los profesionales tratantes deberían considerar evaluar sistemáticamente los efectos de la medicación con el fin de manejar un adecuado ajuste farmacológico que 
considere también la percepción subjetiva del bienestar del paciente y favorezca a una mejor adherencia al tratamiento.

\section{Agradecimientos}

Fuente de apoyo financiero: CONICYT/BECAS-CHILE Postdoctorado en el Extranjero (74140004) y Convenio de Desempeño UTA - MINEDUC.

Agradecemos a Felipe Ponce y Jorge Escudero por su colaboración en este estudio y también las siguientes personas y servicios: Dr. Hugo Sánchez, Dr. Ricardo Alvites, Dr. Andrés Collado, Gladys Coaquira, Vilma Liendo y especialmente al Dr. José Revilla del Hospital Hipólito Unanue de Tacna, Perú; al Dr. Marcio Soto desde Arequipa, Perú; Dr. Fernando Garitano, Dr. Mauricio Peredo y Dra. Mabel Romero del Centro de Rehabilitación y Salud Mental San Juan de Dios; y al Director del Hospital Psiquiátrico de la Caja Nacional de Salud de La Paz, Bolivia. También agradecemos a la Dra. Magdalena Gardilcic, Ester López y Alejandra Lagos de Servicio de Salud de Arica, Chile.

Extendemos además un especial agradecimiento a todos los pacientes y sus familiares que participaron en este proyecto. Finalmente, un agradecimiento especial al Dr. Stephen Gilman del National Institutes of Health (NIH) de Estados Unidos y al Dr. David Williams de la Universidad de Harvard quienes colaboraron en el desarrollo de esta investigación.

\section{Referencias}

Allison, D.B., Mackell, J.A., y McDonnell, D.D. (2003). The impact of weight gain on quality of life among persons with schizophrenia. Psychiatric Services, 54, 565-567.

Awad, A.G., y Hogan, T. P. (1994). Subjective response to neuroleptics and the quality of life: implications for treatment outcome. Acta Psychiatrica Scandinavica, 89, 27-32.

Barnes, T. R. (1989). A rating scale for drug-induced akathisia. British Journal of Psychiatry, 154, 672-676.

Bobes, J., Garcia-Portilla, M. P., Bascaran, M. T., Saiz, P.A., y Bousono, M. (2007). Quality of life in schizophrenic patients. Dialogues in Clinical Neuroscience, 9, 215-26.

Boyer, L., Simeoni, M.C., Loundou, A., D'Amato, T., Reine, G., Lancon, C., y Auquier, P. (2010). The development of the S-QoL 18: a shortened quality of life questionnaire for patients with schizophrenia. Schizophrenia Research, 121, 241-250.

Browne, S. R., Lane, A., Gervin, M., Morris, M., Kinsella, A., Larkin, C., y O'Callaghan, E. (1996). Quality of life in schizophrenia: relationship to socio-demographic factors, symptomatology and tardive dyskinesia. Acta Psychiatrica Scandinavica, 94, 118-124.

Caron, J., Mercier, C., Diaz, P., y Martin, A. (2005). Socio-demographic and clinical predictors of quality of life in patients with schizophrenia or schizo-affective disorder. Psychiatry Research, 137, 203-13.

Górna, K., Jaracz, K., Rybakowski, F., Rybakowski, J. (2008). Determinants of objective and subjective quality of life in first-time-admission schizophrenic patients in Poland: a longitudinal study. Quality of Life Research, 17, 237-47.

Hofer, A., Kemmler, G., Eder, U., Edlinger, M., Hummer, M., Fleischhacker, W.W. (2004). Quality of life in schizophrenia: the impact of psychopathology, attitude toward medication and side effects. Journal of Clinical Psychiatry, 65, 932-9.

Kay, S. R., Fiszbein, A., y Opler, L. (1987). The positive and negative syndrome scale (PANSS) for schizophrenia. Schizophrenia Bulletin, $13,261-76$

Krieger, N. (2007). Why epidemiologists cannot afford to ignore poverty. Epidemiology, 18, 658-663.

Lindström, L.H. (1994). Long-term clinical and Social outcome studies in schizophrenia in relation to the cognitive and emotional side effects of antipsychotic drugs. Acta Psychiatrica Scandinavica, 89, 74-76.

Lund, C., De Silva, M., Plagerson, S., Cooper, S., Chisholm, D., Das, J., y Knapp, M., Patel, V. (2011). Poverty and mental disorders: breaking the cycle in low-income and middle-income countries. Lancet, 378, 1502-14.

Marmot, M., Friel, S., Bell, R., Houweling, T.A., y Taylor, S. (2008). Closing the gap in a generation: health equity through action on the social determinants of health. Lancet, 372, 1661-9.

Marwaha, S., y Jhonson, S. (2004). Schizophrenia and employment: A review. Social Psychiatry and Psychiatric Epidemiology, 39, 337-349.

Meyer, J. M., Nasrallah, H. A., McEvoy, J. P., Goff, D. C., Davis, S. M., Chakos, M., Patel, J. K., Keefe, R. S., Stroup, T. S., y Lieberman, J. A. (2005). The Clinical Antipsychotic Trials of Intervention Effectiveness (CATIE) Schizophrenia Trial: Clinical comparison of subgroups with and without the metabolic syndrome. Schizophrenia Research, 80, 9-18.

Olfson, M., Uttaro, T., Carson, W.H., y Tafesse, E. (2005). Male sexual dysfunction and quality of life in schizophrenia. Journal of Clinical Psychiatry, 66, 331-338.

Peters, D. H., Garg, A., Bloom, G., Walker, D. G., Brieger, W. R., y Rahman, M.H. (2008). Poverty and access to health care in developing countries. Annals of the New York Academy of Sciences, 1136, 161-71.

Pinikahana, J., Hapell, B., Hope, J., y Keks, N. (2002). Quality of life in schizophrenia: A review of the literature from 1995 to 2000. Journal of Psychiatric and Mental Health Nursing, 11, 103-111.

Ritsner, M., Gibel, A., Perelroyzen, G., Kurs, R., Jabarin, M., y Ratner, Y. (2004). Quality of life outcomes of risperidone, olanzapine, and typical antipscyhotics among schizophrenia patients treated in routine clinical practice. A naturalistic comparative study. Journal of Clinical Psychopharmacology, 24, 582-591.

Rudnick, A. (2001). The impact of coping on the relation between symptoms and quality of life in schizophrenia. Psychiatry, 64, 304-308.

Salokangas, R., Honkonen, T., Stengard, E., y Koivisto, A. (2001). To be or not to be married- that is the question of quality of life in men with schizophrenia. Social Psychiatry and Psychiatric Epidemiology, 36, 381-390.

Simpson, G. N., y Angus J. W. S. (1970). A rating scale for extrapiramial side effects. Acta Psychiatrica Scandinavica, 212, 11-19.

Strassnig, M., Brar, J .S., y Ganguli, R. (2003). Body mass index and quality of life in community dwelling patients with schizophrenia. Schizophrenia Research, 62, 73-76.

Wagstaff, A. (2002). Poverty and health sector inequalities. Bulletin of World Health Organization, 80, 97-105.

Wilton, R. (2004). Putting policy into practice? Poverty and people with serious mental illness. Social Science \& Medicine, 58, 25-39.

World Health Organization (WHO). (2013). Report on the Assessment of Mental Health Systems in Latin America and the Caribbean using the World Health Organization Assessment Instrument for Mental Health Systems (WHO-AIMS). Recuperado desde http://www.who.int/mental_health/evidence/WHO-AIMS/en/

Xiang, Y., Wang, C. Y., Wang, Y., Chiu, H. F., Zhao, J. P., Chen, Q., Chan, S. S., Lee, E. H., y Ungvari, G.S. (2010). Socio-demographic and clinical determinants of quality of life in Chinese patients with schizophrenia: a prospective study. Quality of Life Research, 19, 317-322. 